\title{
General Relativity from Scattering Amplitudes
}

\author{
N. E. J. Bjerrum-Bohr, ${ }^{1}$ Poul H. Damgaard, ${ }^{1}$ Guido Festuccia, ${ }^{2}$ Ludovic Planté, ${ }^{3}$ and Pierre Vanhove ${ }^{4,5}$ \\ ${ }^{1}$ Niels Bohr International Academy and Discovery Center, The Niels Bohr Institute, \\ Blegdamsvej 17, DK-2100 Copenhagen $\emptyset$, Denmark \\ ${ }^{2}$ Department of Physics and Astronomy, Theoretical Physics, Angströmlaboratoriet, \\ Lägerhyddsvägen 1, Box 51675120 Uppsala, Sweden \\ ${ }^{3}$ Ministère de l'économie et des finances, Direction générale des entreprises, 94200 Ivry-sur-Seine, France \\ ${ }^{4}$ Institut de Physique Théorique, Université Paris-Saclay, CEA, CNRS, F-91191 Gif-sur-Yvette Cedex, France \\ ${ }^{5}$ National Research University Higher School of Economics, 123592 Moscow, Russian Federation
}

(Received 21 June 2018; published 25 October 2018)

\begin{abstract}
Weoutline the program to apply modern quantum field theory methods to calculate observables in classical general relativity through a truncation to classical terms of the multigraviton, two-body, on-shell scattering amplitudes between massive fields. Since only long-distance interactions corresponding to nonanalytic pieces need to be included, unitarity cuts provide substantial simplifications for both postNewtonian and post-Minkowskian expansions. We illustrate this quantum field theoretic approach to classical general relativity by computing the interaction potentials to second order in the post-Newtonian expansion, as well as the scattering functions for two massive objects to second order in the postMinkowskian expansion. We also derive an all-order exact result for gravitational light-by-light scattering.
\end{abstract}

DOI: 10.1103/PhysRevLett.121.171601

Today it is universally accepted that classical general relativity can be understood as the $\hbar \rightarrow 0$ limit of a quantum mechanical path integral with an action that, minimally, includes the Einstein-Hilbert term. It describes gravitational interactions in terms of exchanges and interactions of spin-2 gravitons with themselves (and with matter) [1,2]. The language of effective field theory encompasses this viewpoint, and it shows that a long-distance quantum field theoretic description of gravity is well defined order by order in a derivative expansion [3,4]. Quantum mechanics thus teaches us that we should expect classical general relativity to be augmented by higher-derivative terms. More remarkably, what would ordinarily be a quantum mechanical loop expansion contains pieces at an arbitrarily high order that are entirely classical [5,6]. A subtle cancellation of factors of $\hbar$ is at work here. This leads to the radical conclusion that one can define classical general relativity perturbatively through the loop expansion. Then $\hbar$ plays a role only at intermediary steps, a dimensional regulator that is unrelated to the classical physics the path integral describes.

For the loop expansion, central tools have been the unitarity methods [7] that reproduce those parts of loop amplitudes that are "cut constructable," i.e., all nonanalytic terms of the amplitudes. This amounts to an enormous

Published by the American Physical Society under the terms of the Creative Commons Attribution 4.0 International license. Further distribution of this work must maintain attribution to the author(s) and the published article's title, journal citation, and DOI. Funded by SCOAP ${ }^{3}$. simplification, and most of today's amplitude computations for the Standard Model of particle physics would not have been possible without this method. In classical gravity, the long-distance terms we seek are precisely of such a nonanalytic kind, being functions of the dimensionless ratio $m / \sqrt{-q^{2}}$, where $m$ is a massive probe, and $q^{\mu}$ describes a suitably defined momentum transfer [4]. This leads to the proposal that these modern methods be used to compute post-Newtonian and post-Minkowskian perturbation theory of general relativity for astrophysical processes such as binary mergers. This has acquired new urgency due to the recent observations of gravitational waves emitted during such inspirals.

While the framework for classical general relativity as described above would involve all possible interaction terms in the Lagrangian, ordered according to a derivative expansion, one can always choose to retain only the Einstein-Hilbert action. Quantum mechanically this is inconsistent, but for the purpose of extracting only classical results from that action, it is a perfectly valid truncation. This scheme relies on a separation of the long-distance (infrared) and short-distance (ultraviolet) contributions in the scattering amplitudes in quantum field theory. We will follow that strategy here, but one may apply the same amplitude methods to actions that contain, already at the classical level, higher-derivative terms as well. In the future, this may be used to put better observational bounds on such new couplings.

In Ref. [8], Damour proposed a new approach for converting classical scattering amplitudes into the 
effective-one-body Hamiltonian of two gravitationally interacting bodies. In this Letter, we take a different route and we show how scattering amplitude methods, which build on the probabilistic nature of quantum mechanics, may be used to derive classical results in gravity. We show how tree-level massless emission from massive classical sources arises from quantum multiloop amplitudes, thus providing an all-order argument extending the original observations in Ref. [6]. We apply this method to derive the scattering angle between two masses to second postMinkowskian order using the eikonal method.

We start with the Einstein-Hilbert action coupled to a scalar field $\phi$ :

$\mathcal{S}=\int d^{4} x \sqrt{-g}\left(\frac{1}{16 \pi G} R+\frac{1}{2} g^{\mu \nu} \partial_{\mu} \phi \partial_{\nu} \phi-\frac{m^{2}}{2} \phi^{2}\right)$.

Here $R$ is the curvature and $g_{\mu \nu}$ is the metric, defined as the sum of a flat Minkowski component $\eta_{\mu \nu}$ and a perturbation $\kappa h_{\mu \nu}$ with $\kappa \equiv \sqrt{32 \pi G}$. It is coupled to the scalar stressenergy tensor $T_{\mu \nu} \equiv \partial_{\mu} \phi \partial_{\nu} \phi-\left(\eta_{\mu \nu} / 2\right)\left(\partial^{\rho} \phi \partial_{\rho} \phi-m^{2} \phi^{2}\right)$.

Scalar triangle integrals [9] are what reduces the oneloop, two-graviton scattering amplitude to classical general relativity $[4,10-12]$ in four dimensions. For the longdistance contributions these are the integrals that produce the tree-like structures one intuitively associates with classical general relativity. To see this, consider first the triangle integral of one massive and two massless propagators,

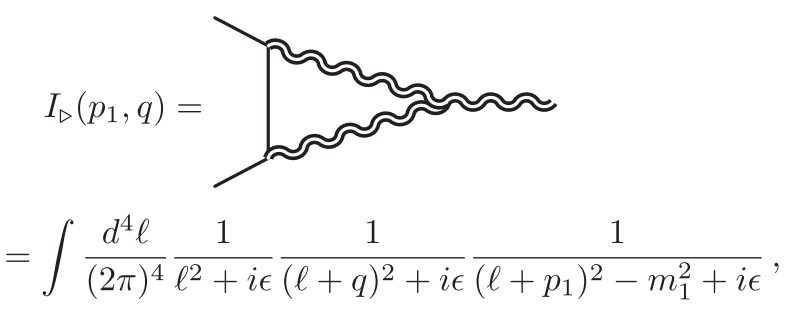

with $p_{1}=(E, \vec{q} / 2), p_{2}=(E,-\vec{q} / 2)$ and $q \equiv p_{1}-p_{1}^{\prime}=(0, \vec{q})$, and $E=\sqrt{m_{1}^{2}+\vec{q}^{2} / 4}$, and we work with the mostly negative metric (+---). The curly lines are for massless fields and the left solid line is for a particle of incoming momentum $p_{1}$, outgoing momentum $p_{1}^{\prime}$, and mass $p_{1}^{2}=p_{1}^{\prime 2}=m_{1}^{2}$.

In the large mass approximation, we focus on the region $|\vec{\ell}| \ll m_{1}$, and we have $\left(\ell+p_{1}\right)^{2}-m_{1}^{2}=\ell^{2}+2 \ell p_{1} \simeq 2 m_{1} \ell_{0}$; therefore the integral reduces in that limit to

$$
\frac{1}{2 m_{1}} \int \frac{d^{4} \ell}{(2 \pi)^{4}} \frac{1}{\ell^{2}+i \epsilon} \frac{1}{(\ell+q)^{2}+i \epsilon} \frac{1}{\ell_{0}+i \epsilon} .
$$

We perform the $\ell_{0}$ integration by closing the contour of integration in the upper half-plane to get

$$
\int_{|\vec{e}| \ll m} \frac{d^{3} \vec{\ell}}{(2 \pi)^{3}} \frac{i}{4 m} \frac{1}{\vec{\ell}^{2}} \frac{1}{(\vec{\ell}+q)^{2}}=-\frac{i}{32 m|\vec{q}|} .
$$

This result can be obtained by performing the large mass expansion of the exact expression for the triangle integral as shown in the Supplemental Material [13].

In Eq. (4) we recognize the three-dimensional integral of two static sources localized at different positions, represented as shaded blobs, and emitting massless fields

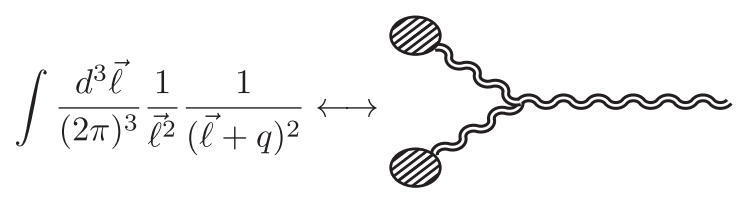

Below we show how this allows us to recover the first postNewtonian correction to the Schwarzschild metric from quantum loops. We now explain how the classical part emerges from higher-loop triangle graphs, starting with two-loop triangles
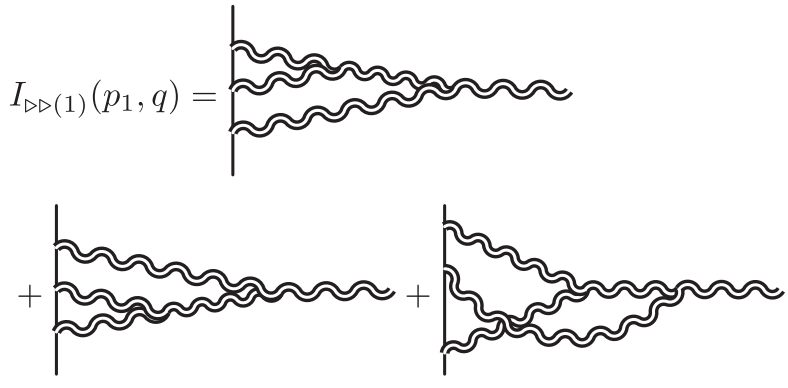

In the large mass limit $\left|\vec{\ell}_{i}\right| \ll m_{1}$ for $i=1,2,3$ and approximating $\left(\ell_{i}+p_{1}\right)^{2}-m_{1}^{2} \simeq 2 \ell_{i} p_{1} \simeq 2 m_{1} \ell_{i}^{0}$, the integral reduces in that limit to

$$
\begin{aligned}
I_{\triangleright \triangleright(1)} & \left(p_{1}, q\right) \\
= & -\int \prod_{i=1}^{3} \frac{d^{4} \ell_{i}}{(2 \pi)^{4}} \frac{1}{\ell_{i}^{2}+i \epsilon} \\
& \times \frac{(2 \pi)^{3} \delta^{(3)}\left(\sum_{i=1}^{3} \vec{\ell}_{i}+\vec{q}\right)}{\left(\ell_{1}+q\right)^{2}+i \epsilon} \times 2 \pi \delta\left(\ell_{1}^{0}+\ell_{2}^{0}+\ell_{3}^{0}\right) \\
& \times\left(\frac{1}{2 m_{1} \ell_{1}^{0}+i \epsilon} \frac{1}{2 m_{1} \ell_{2}^{0}-i \epsilon}+\frac{1}{2 m_{1} \ell_{3}^{0}+i \epsilon} \frac{1}{2 m_{1} \ell_{1}^{0}-i \epsilon}\right. \\
& \left.+\frac{1}{2 m_{1} \ell_{3}^{0}+i \epsilon} \frac{1}{2 m_{1} \ell_{2}^{0}-i \epsilon}\right) .
\end{aligned}
$$

We note that 


$$
\begin{aligned}
\delta\left(\ell_{1}^{0}\right. & \left.+\ell_{2}^{0}+\ell_{3}^{0}\right)\left(\frac{1}{2 m_{1} \ell_{1}^{0}+i \epsilon} \frac{1}{2 m_{1} \ell_{2}^{0}-i \epsilon}\right. \\
& \left.+\frac{1}{2 m_{1} \ell_{3}^{0}+i \epsilon} \frac{1}{2 m_{1} \ell_{1}^{0}-i \epsilon}+\frac{1}{2 m_{1} \ell_{3}^{0}+i \epsilon} \frac{1}{2 m_{1} \ell_{2}^{0}-i \epsilon}\right) \\
= & 0+O(\epsilon),
\end{aligned}
$$

so that only the $\ell_{0}$ residue at $2 m_{1} \ell^{0}= \pm i \epsilon$ contributes, giving

$$
\begin{aligned}
I_{\triangleright \triangleright(1)}\left(p_{1}, q\right)= & \frac{i}{4 m_{1}^{2}} \int \frac{d^{3} \vec{\ell}_{1}}{(2 \pi)^{3}} \frac{d^{3} \vec{\ell}_{2}}{(2 \pi)^{3}} \frac{1}{\vec{\ell}_{1}^{2}} \frac{1}{\vec{\ell}_{2}^{2}} \\
& \times \frac{1}{\left(\vec{\ell}_{1}+\vec{\ell}_{2}+\vec{q}\right)^{2}} \frac{1}{\left(\vec{\ell}_{1}+\vec{q}\right)^{2}} .
\end{aligned}
$$

We now consider the large mass expansion of the graph

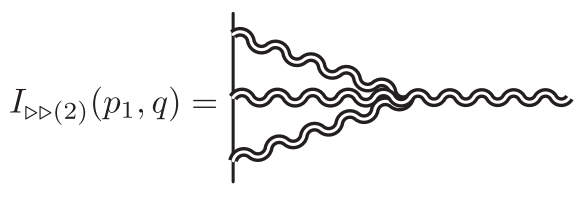

which, to leading order, reads

$$
\begin{aligned}
& I_{\triangleright \triangleright(d)}\left(p_{1}, q\right) \\
&=-\frac{1}{3} \int \prod_{i=1}^{3}\left(\frac{d^{4} \ell_{i}}{(2 \pi)^{4}} \frac{1}{\ell_{i}^{2}+i \epsilon}\right) \\
& \quad \times(2 \pi)^{3} \delta^{(3)}\left(\ell_{1}+\ell_{2}+\ell_{3}+q\right) \\
& \quad \times 2 \pi \delta\left(\ell_{1}^{0}+\ell_{2}^{0}+\ell_{3}^{0}\right)\left(\frac{1}{2 m_{1} \ell_{1}^{0}+i \epsilon} \frac{1}{2 m_{1} \ell_{2}^{0}-i \epsilon}\right. \\
&\left.+\frac{1}{2 m_{1} \ell_{3}^{0}+i \epsilon} \frac{1}{2 m_{1} \ell_{1}^{0}-i \epsilon}+\frac{1}{2 m_{1} \ell_{3}^{0}+i \epsilon} \frac{1}{2 m_{1} \ell_{2}^{0}-i \epsilon}\right),
\end{aligned}
$$

and evaluates to

$$
I_{\triangleright \triangleright(2)}\left(p_{1}, q\right)=\frac{1}{12 m_{1}^{2}} \int \frac{d^{3} \vec{\ell}_{1}}{(2 \pi)^{3}} \frac{d^{3} \vec{\ell}_{2}}{(2 \pi)^{3}} \frac{1}{\vec{\ell}_{1}^{2}} \frac{1}{\vec{\ell}_{2}^{2}} \frac{1}{\left(\vec{\ell}_{1}+\vec{\ell}_{2}+\vec{q}\right)^{2}} .
$$

Equations (9) and (12) are precisely the coupling of three static sources to a massless tree amplitude

$$
I_{\triangleright \triangleright(1)}\left(p_{1}, q\right), I_{\triangleright \triangleright(2)}\left(p_{1}, q\right) \leftrightarrow
$$

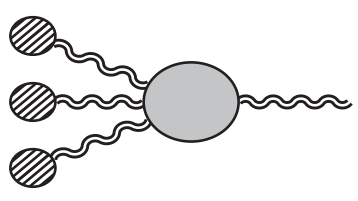

A generalization of the identity [Eq. (8)] implies that the sum of all the permutation of $n$ massless propagators connected to a massive scalar line results in the coupling of classical sources to multileg tree amplitudes [20,21]. The same conclusion applies to massive particles with spin as we will demonstrate elsewhere.

This analysis applies directly to the computation of an off-shell quantity such as the metric itself. Consider the absorption of a graviton

$$
\begin{aligned}
& \left\langle p_{2}\left|T_{\mu \nu}\right| p_{1}\right\rangle \\
& =\frac{-i}{2 m_{1}} \int \frac{d^{4} \ell}{(2 \pi)^{4}} \frac{P_{\rho \sigma, \alpha \beta}}{\ell^{2}+i \epsilon} \frac{P_{\kappa \lambda, \gamma \delta}}{(\ell+q)^{2}+i \epsilon} \\
& \times \frac{\tau_{1}^{\rho \sigma}\left(p_{1}-\ell, p_{1}\right) \tau_{1}^{\kappa \lambda}\left(p_{1}-\ell, p_{2}\right) \tau_{3 \mu \nu}{ }^{\alpha \beta, \gamma \delta}(\ell, \ell-q)}{\left(\ell+p_{1}\right)^{2}-m_{1}^{2}+i \epsilon},
\end{aligned}
$$

where $\tau_{1}$ is the vertex for the coupling of one graviton to a scalar given in Ref. [[22], Eq. (72)], $\tau_{3}$ is the three graviton vertex given in Ref. [[22], Eq. (73)], and $P_{\mu \nu, \rho \sigma}$ is the projection operator given in Ref [[22], Eq. (30)]. In the large $m$ limit $|q| / m \ll 1$ projects the integral on the 00component of the scalar vertex $\tau_{1}^{\mu \nu}\left(p_{1}-\ell, p_{1}\right) \simeq i \kappa m_{1}^{2} \delta_{0}^{\mu} \delta_{0}^{\nu}$. Focusing on the 00 component, we have in this limit [21]

$$
\begin{aligned}
\left\langle p_{2}\left|T_{00}\right| p_{1}\right\rangle & \simeq 4 i \pi G m_{1}^{3} \int \frac{d^{4} \ell}{(2 \pi)^{4}}\left(\frac{3}{8} \vec{q}^{2}-\frac{3}{2} \vec{\ell}^{2}\right) \\
& \times \frac{1}{\left(\ell^{2}+i \epsilon\right)\left[(\ell+q)^{2}+i \epsilon\right]\left[\left(\ell+p_{1}\right)^{2}-m_{1}^{2}+i \epsilon\right]} \\
& =\frac{3 \kappa^{2} m^{3}}{128}|\vec{q}|,
\end{aligned}
$$

where we used the result of the previous section to evaluate the triangle integral. This reproduces the classical first postNewtonian contribution to the 00-component of the Schwarzschild metric evaluated in Ref. [22]. It also immediately shows how to relate a conventional Feynman-diagram evaluation with the computation of Duff [23] who derived such tree-like structures from classical sources.

Scalar interaction potentials.-For the classical terms we need only the graviton cuts, and instead of computing classes of diagrams, we apply the unitarity method directly to get the on-shell scattering amplitudes initiated in Ref. [10] and further developed in [11,12]. We first consider the scattering of two scalars of masses $m_{1}$ and $m_{2}$, respectively. At one-loop order this entails a twograviton cut of a massive scalar four-point amplitude. We have shown that classical terms arise from topologies with loops solely entering as triangles that include the massive states. When we glue together the two on-shell scattering amplitudes, we thus discard all terms that do not correspond to such topologies. Rational terms are not needed, as they correspond to ultralocal terms of no relevance for the long-distance interaction potentials. 
We first recall the classical tree-level result from the onegraviton exchange

$$
\begin{aligned}
\mathcal{M}_{1} & =\approx \\
& =-\frac{16 \pi G}{q^{2}}\left(m_{1}^{2} m_{2}^{2}-2\left(p_{1} \cdot p_{2}\right)^{2}-\left(p_{1} \cdot p_{2}\right) q^{2}\right),
\end{aligned}
$$

where incoming momenta are $p_{1}$ and $p_{2}$ and $p_{1}^{2}=p_{1}^{\prime 2}=m_{1}^{2}$, $p_{2}^{2}=p_{2}^{\prime 2}=m_{2}^{2}$ and the momentum transfer $q=p_{1}-p_{1}^{\prime}=$ $-p_{2}+p_{2}^{\prime}$.

The two-graviton interaction is clearly a one-loop amplitude that can be constructed using the on-shell unitarity method [10-12]. The previous analysis shows that the classical piece is contained in the triangle graphs

$$
\mathcal{M}_{2}=-i(8 \pi G)^{2}\left(\frac{c\left(m_{1}, m_{2}\right) I_{\triangleright}\left(p_{1}, q\right)}{\left(q^{2}-4 m_{1}^{2}\right)^{2}}+\frac{c\left(m_{2}, m_{1}\right) I_{\triangleright}\left(p_{2},-q\right)}{\left(q^{2}-4 m_{2}^{2}\right)^{2}}\right),
$$

with, for the interaction between two massive scalars,

$$
\begin{aligned}
c\left(m_{1}, m_{2}\right)= & \left(q^{2}\right)^{5}+\left(q^{2}\right)^{4}\left(6 p_{1} \cdot p_{2}-10 m_{1}^{2}\right)+\left(q^{2}\right)^{3}\left(12\left(p_{1} \cdot p_{2}\right)^{2}-60 m_{1}^{2} p_{1} \cdot p_{2}-2 m_{1}^{2} m_{2}^{2}+30 m_{1}^{4}\right) \\
& -\left(q^{2}\right)^{2}\left(120 m_{1}^{2}\left(p_{1} \cdot p_{2}\right)^{2}-180 m_{1}^{4} p_{1} \cdot p_{2}-20 m_{1}^{4} m_{2}^{2}+20 m_{1}^{6}\right) \\
& +q^{2}\left(360 m_{1}^{4}\left(p_{1} \cdot p_{2}\right)^{2}-120 m_{1}^{6} p_{1} \cdot p_{2}-4 m_{1}^{6}\left(m_{1}^{2}+15 m_{2}^{2}\right)\right)+48 m_{1}^{8} m_{2}^{2}-240 m_{1}^{6}\left(p_{1} \cdot p_{2}\right)^{2} .
\end{aligned}
$$

At leading order in $q^{2}$, using the result of Eq. (4), the two gravitons exchange simplifies to just, in agreement with Ref. [[24], Eq. (3.26)] and Refs. [10,25],

$\mathcal{M}_{2}=\frac{6 \pi^{2} G^{2}}{|\vec{q}|}\left(m_{1}+m_{2}\right)\left[5\left(p_{1} \cdot p_{2}\right)^{2}-m_{1}^{2} m_{2}^{2}\right]+O(|\vec{q}|)$.

Note the systematics of this expansion. The Einstein metric is expanded perturbatively, and all physical momenta are provided at infinity. Contractions of momenta are performed with respect to the flat-space Minkowski metric only, and no reference is made to space-time coordinates. This is a gauge invariant expression for the classical scattering amplitude in a plane wave basis that is independent of coordinate choices (and gauge choices). To derive a classical nonrelativistic potential, we need to choose coordinates: we Fourier transform the gauge invariant momentum-space scattering amplitude. This introduces coordinate dependence even in theories such as quantum electrodynamics. Moreover, just as in quantum electrodynamics, we must also be careful in keeping subleading terms of this Fourier transform and thus expand in $q^{0}$ consistently. This forces us to keep velocity-dependent terms in the energy that are of the same order as the naively defined static potential. One easily checks that the overall sign of the amplitudes in Eqs. (16) and (19) are precisely the ones required for an attractive force.

The result of this procedure has been well documented elsewhere, starting with the pioneering observations of Iwasaki [5], and later reproduced in different coordinates in Refs. $[10,26]$. Although we are unable to reproduce the individual contributions in Ref. [[5], Eqs. (A.1.4)-(A.1.6)] our final result for the interaction energy is to this order:

$$
\begin{aligned}
H= & \frac{\vec{p}_{1}^{2}}{2 m_{1}}+\frac{\vec{p}_{2}^{2}}{2 m_{2}}-\frac{\vec{p}_{1}^{4}}{8 m_{1}^{3}}-\frac{\vec{p}_{2}^{4}}{8 m_{2}^{3}} \\
& -\frac{G m_{1} m_{2}}{r}-\frac{G^{2} m_{1} m_{2}\left(m_{1}+m_{2}\right)}{2 r^{2}} \\
& -\frac{G m_{1} m_{2}}{2 r}\left(\frac{3 \vec{p}_{1}^{2}}{m_{1}^{2}}+\frac{3 \vec{p}_{2}^{2}}{m_{2}^{2}}-\frac{7 \vec{p}_{1} \cdot \vec{p}_{2}}{m_{1} m_{2}}-\frac{\left(\vec{p}_{1} \cdot \vec{r}\right)\left(\vec{p}_{2} \cdot \vec{r}\right)}{m_{1} m_{2} r^{2}}\right),
\end{aligned}
$$

which precisely leads to the celebrated Einstein-InfeldHoffmann equations of motion. It is crucial to correctly perform the subtraction of the iterated tree-level Born term in order to achieve this.

The post-Minkowskian expansion.-The scattering problem of general relativity can be treated in a fully relativistic manner, without a truncated expansion in velocities. To this end, we consider here the full relativistic scattering amplitude and expand in Newton's constant $G$ only. For the scalarscalar case we thus return to the complete classical one-loop result [Eq. (17)]. The conventional Born-expansion expression that is used to derive the quantum mechanical cross section is not appropriate here, even if we keep only the classical part of the amplitude. That expression for the cross section is based on incoming plane waves, and will not match the corresponding classical cross section beyond the leading tree-level term. In fact, even the classical cross section is unlikely to be of any interest observationally. So a more meaningful approach is to use the classical scattering amplitude to compute the scattering angle of two masses colliding with a given impact parameter $b$.

We use the eikonal approach to derive the relationship between small scattering angle $\theta$ and impact parameter $b$. 
Generalizing the analysis of Refs. [27,28] (please refer to the Supplemental Material [13] for some details on the eikonal method to one-loop order) to the case of two scalars of masses $m_{1}$ and $m_{2}$, we focus on the high-energy regime $s, t$ large, and $t / s$ small. Note that in addition to expanding in $G$, we are also expanding the full result [Eq. (17)] in $q^{2}$, and truncating already at next-to-leading order. We go to the center of mass frame and define $p \equiv\left|\vec{p}_{1}\right|=\left|\vec{p}_{2}\right|$. The impact parameter is defined by a two-dimensional vector $\vec{b}$ in the plane of scattering orthogonal to $\vec{p}_{1}=-\vec{p}_{2}$, with $b \equiv|\vec{b}|$. In the eikonal limit we find the exponentiated relationship between the scattering amplitude

$$
M(\vec{b}) \equiv \int d^{2} \vec{q} e^{-i \vec{q} \cdot \vec{b}} M(\vec{q}),
$$

and scattering function $\chi(b)$ to be

$$
M(\vec{b})=4 p\left(E_{1}+E_{2}\right)\left(e^{i \chi(\vec{b})}-1\right) .
$$

In order to compare with the first computation of postMinkowskian scattering to order $G^{2}$ [29], we introduce new kinematical variables $M^{2} \equiv s, \hat{M}^{2} \equiv M^{2}-m_{1}^{2}-m_{2}^{2}$. We go to the center of mass frame where $p^{2}=$ $\left(\hat{M}^{4}-4 m_{1}^{2} m_{2}^{2}\right) / 4 M^{2}$. In terms of the scattering angle $\theta$ we have $t \equiv q^{2}=\left[\left(\hat{M}^{4}-4 m_{1}^{2} m_{2}^{2}\right) \sin ^{2}(\theta / 2)\right] / M^{2}$, and $4 p\left(E_{1}+E_{2}\right)=2 \sqrt{\hat{M}^{4}-4 m_{1}^{2} m_{2}^{2}}$. Keeping, consistently, only the leading order in $q^{2}$ of the one-loop amplitude [Eq. (21)], we obtain

$$
2 \sin (\theta / 2)=\frac{-2 M}{\sqrt{\hat{M}^{4}-4 m_{1}^{2} m_{2}^{2}}} \frac{\partial}{\partial b}\left[\chi_{1}(b)+\chi_{2}(b)\right],
$$

where $\chi_{1}(b)$ and $\chi_{2}(b)$ are the tree-level and one-loop scattering functions given respectively by the Fourier transform of the scattering amplitudes

$$
\chi_{i}(b)=\frac{1}{2 \sqrt{\hat{M}^{4}-4 m_{1}^{2} m_{2}^{2}}} \int \frac{d^{2} \vec{q}}{(2 \pi)^{2}} e^{-i \vec{q} \cdot \vec{b}} \mathcal{M}_{i}(\vec{q}) .
$$

At leading order in $q^{2}$ the tree-level and one-loop amplitudes in Eqs. (16) and (19) read

$$
\begin{aligned}
& \mathcal{M}_{1}(\vec{q})=\frac{8 \pi G}{\vec{q}^{2}}\left(\hat{M}^{4}-2 m_{1}^{2} m_{2}^{2}\right), \\
& \mathcal{M}_{2}(\vec{q})=\frac{3 \pi^{2} G^{2}}{2|\vec{q}|}\left(m_{1}+m_{2}\right)\left(5 \hat{M}^{4}-4 m_{1}^{2} m_{2}^{2}\right),
\end{aligned}
$$

where higher order terms in $q^{2}$ correspond in position space to quantum corrections. Only the triangle contribution contribute to the one-loop scattering function because the contributions from the boxes and cross-boxes contributed to the exponentiation of the tree-level amplitude $[28,30]$. The Fourier transform around two dimensions is computed using

$$
\mu^{2-D} \int \frac{d^{D} q}{(2 \pi)^{D}} e^{-i \vec{q} \cdot \vec{b}}|\vec{q}|^{\alpha}=\frac{(2 \pi \mu)^{2-D}}{4 \pi}\left(\frac{2}{b}\right)^{\alpha+D} \frac{\Gamma \frac{(\alpha+D}{2)}}{\Gamma \frac{(2-\alpha-D}{2)}} .
$$

The scattering functions then read

$$
\begin{aligned}
& \chi_{1}(b)=2 G \frac{\hat{M}^{4}-2 m_{1}^{2} m_{2}^{2}}{\sqrt{\hat{M}^{4}-4 m_{1}^{2} m_{2}^{2}}}\left(\frac{1}{d-2}-\log (\pi \mu b)-\gamma_{E}\right), \\
& \chi_{2}(b)=\frac{3 \pi G^{2}}{8 \sqrt{\hat{M}^{4}-4 m_{1}^{2} m_{2}^{2}}} \frac{m_{1}+m_{2}}{b}\left(5 \hat{M}^{4}-4 m_{1}^{2} m_{2}^{2}\right),
\end{aligned}
$$

where $\mu$ is a regularization scale. The scattering angle to this order reads

$$
\begin{aligned}
2 \sin \left(\frac{\theta}{2}\right)= & \frac{4 G M}{b}\left(\frac{\hat{M}^{4}-2 m_{1}^{2} m_{2}^{2}}{\hat{M}^{4}-4 m_{1}^{2} m_{2}^{2}}\right. \\
& \left.+\frac{3 \pi}{16} \frac{G\left(m_{1}+m_{2}\right)}{b} \frac{5 \hat{M}^{4}-4 m_{1}^{2} m_{2}^{2}}{\hat{M}^{4}-4 m_{1}^{2} m_{2}^{2}}\right) .
\end{aligned}
$$

This result agrees with the expression found by Westpfahl [29] who explicitly solved the Einstein equations to this order in $G$ and in the same limit of small scattering angle. We find the present approach to be superior in efficiency, and very easily generalizable to higher orders in $G$.

Taking the massless limit $m_{2}=0$ and approximating $2 \sin (\theta / 2) \simeq \theta$, we recover the classical bending angle of light $\theta=\left(4 G m_{1} / b\right)+(15 \pi / 4)\left(G^{2} m_{1}^{2} / b^{2}\right)$, including its first nontrivial correction in $G$, in agreement with Ref. [[31], §101]. We have additionally computed the full expression for the classical part of the scalar-fermion (spin $1 / 2$ ) amplitude up to and including one-loop order, but do not display the results here for lack of space. We stress that the small-angle scattering formula derived above is based on only a small amount of the information contained in the full one-loop scattering amplitude [Eq. (17)].

Light-by-light scattering in general relativity.-Photonphoton scattering is particularly interesting, as our analysis will show how to derive an exact result in general relativity. As explained above, classical contributions from loop diagrams require the presence of massive triangles in the loops. For photon-photon scattering, there are no such contributions to any order in the expansion, and we conclude that photon-photon scattering in general relativity is tree-level exact, as follows: 


$$
\begin{aligned}
& \left.M_{\gamma \gamma}=\right\}^{\approx} \approx\{+\cdots\} \\
& -8 \pi G \frac{2 \operatorname{tr}\left(f_{1} f_{2} f_{3} f_{4}\right)+2 \operatorname{tr}\left(f_{1} f_{3} f_{4} f_{2}\right)-\operatorname{tr}\left(f_{1} f_{2}\right) \operatorname{tr}\left(f_{3} f_{4}\right)}{\left(p_{1}-p_{2}\right)^{2}} \\
& -8 \pi G \frac{2 \operatorname{tr}\left(f_{1} f_{4} f_{3} f_{2}\right)+2 \operatorname{tr}\left(f_{1} f_{3} f_{2} f_{4}\right)-\operatorname{tr}\left(f_{1} f_{4}\right) \operatorname{tr}\left(f_{2} f_{3}\right)}{\left(p_{1}+p_{4}\right)^{2}} \\
& -8 \pi G \frac{2 \operatorname{tr}\left(f_{1} f_{3} f_{4} f_{2}\right)+2 \operatorname{tr}\left(f_{1} f_{3} f_{2} f_{4}\right)-\operatorname{tr}\left(f_{1} f_{3}\right) \operatorname{tr}\left(f_{2} f_{4}\right)}{\left(p_{1}-p_{3}\right)^{2}},
\end{aligned}
$$

where the traces are evaluated over the Lorentz indices and $f_{i}^{\mu \nu}=\epsilon_{i}^{\mu} p_{i}^{\nu}-\epsilon_{i}^{\nu} p_{i}^{\mu}$ are the field strength of the photon fields. When considering polarized photons, it is immediate to check that this amplitude is nonvanishing only for scattering of photons of opposite helicity as no force is expected between photons of the same helicity. Similarly the force between parallel photons vanishes [32].

Conclusion.-We have explicitly shown how loops of the Feynman diagram expansion become equal to the treelike structures coupled to classical sources thus demystifying the appearance of loop diagrams in classical gravity, and, at the same time, linking the source-based method directly to conventional Feynman diagrams. Interestingly, the manner in which the $\ell^{0}$ integrations conspire to leave tree-like structures from loops of triangle graphs also forms the precise bridge to classical general relativity computations based on the world-line formulation (see, e.g., Refs. [33-39]).

Enormous simplifications occur when computing what corresponds to on-shell quantities, based on the unitarity method [7]. Nonanalytic terms [4] involving powers of $m / \sqrt{-q^{2}}$ produce the long-distance classical contributions from the loops. By the rules of unitarity cuts, we can reconstruct these nonanalytic pieces by gluing tree-level amplitudes together while summing over physical states of the graviton legs only [10-12,24,25,30].

Scalar interaction potentials form the backbone of gravitational wave computations for binary mergers. The fact that the unitarity method provides these results straightforwardly provides hope that this is the beginning of a new approach to both post-Newtonian and postMinkowskian calculations in general relativity, including those relevant for the physics of gravitational waves. Since the method applies to the general effective field theory of gravity, this opens up a way to constrain terms beyond the Einstein-Hilbert action that may affect the observational signal of gravitational waves.

We thank John Joseph Carrasco, Thibault Damour, Barak Kol, Rafael Porto, Dennis Rätzel, and Ira Rothstein for useful comments on the manuscript. N.E. J.B and P.H.D. are supported in part by the
Danish National Research Foundation (DNRF91). P. V. is partially supported by "Amplitudes" ANR-17CE31-0001-01, and Laboratory of Mirror Symmetry National Research University Higher School of Economics, Russian Federation Government Grant No. agreement No. 14.641.31.0001. G. F. is supported by the ERC STG Grant No. 639220.

[1] R. P. Feynman, Acta Phys. Pol. 24, 697 (1963).

[2] B.S. DeWitt, Phys. Rev. 160, 1113 (1967); 162, 1195 (1967); 162, 1239 (1967).

[3] S. Weinberg, in General Relativity: An Einstein Centenary Survey, edited by S. W. Hawking and W. Israel (Cambridge University Press, Cambridge, England, 1979).

[4] J. F. Donoghue, Phys. Rev. Lett. 72, 2996 (1994); Phys. Rev. D 50, 3874 (1994).

[5] Y. Iwasaki, Prog. Theor. Phys. 46, 1587 (1971).

[6] B. R. Holstein and J. F. Donoghue, Phys. Rev. Lett. 93, 201602 (2004).

[7] Z. Bern, L. J. Dixon, D. C. Dunbar, and D. A. Kosower, Nucl. Phys. B425, 217 (1994); B435, 59 (1995).

[8] T. Damour, Phys. Rev. D 94, 104015 (2016); 97, 044038 (2018).

[9] Z. Bern, L. J. Dixon, and D. A. Kosower, Phys. Lett. B 302 , 299 (1993); 318, 649(E) (1993).

[10] D. Neill and I. Z. Rothstein, Nucl. Phys. B877, 177 (2013).

[11] N. E. J. Bjerrum-Bohr, J. F. Donoghue, and P. Vanhove, J. High Energy Phys. 02 (2014) 111.

[12] V. Vaidya, Phys. Rev. D 91, 024017 (2015).

[13] See Supplemental Material at http://link.aps.org/ supplemental/10.1103/PhysRevLett.121.171601 for some details on the eikonal method to one-loop order and the evaluation of scalar triangle integral, which includes Refs. [14-19].

[14] A. Luna, S. Melville, S. G. Naculich, and C. D. White, J. High Energy Phys. 01 (2017) 052.

[15] A. K. Collado, P. Di Vecchia, R. Russo, and S. Thomas, arXiv:1807.04588.

[16] M. E. Peskin and D. V. Schroeder, An Introduction to quantum field theory (Addison-Wesley, Reading, USA, 1995).

[17] C. Cheung, I. Z. Rothstein, and M.P. Solon, arXiv: 1808.02489.

[18] P. Vanhove, Proc. Symp. Pure Math. 88, 161 (2014).

[19] D. Zagier, in The Dilogarithm Function, in Frontiers in Number Theory, Physics and Geometry II, edited by $\mathrm{P}$. Cartier, B. Julia, P. Moussa, and P. Vanhove (SpringerVerlag, Berlin, Heidelberg, New York, 2006), pp. 3-65.

[20] L. Planté, Ph.D. thesis, Université Paris-Saclay, 2016.

[21] J. Galusha, M.Sc. thesis, Niels Bohr Institute, Copenhagen University, 2015.

[22] N. E. J. Bjerrum-Bohr, J. F. Donoghue, and B. R. Holstein, Phys. Rev. D 68, 084005 (2003).

[23] M. J. Duff, Phys. Rev. D 7, 2317 (1973).

[24] A. Guevara, arXiv:1706.02314.

[25] F. Cachazo and A. Guevara, arXiv:1705.10262.

[26] B. R. Holstein and A. Ross, arXiv:0802.0716.

[27] D. N. Kabat and M. Ortiz, Nucl. Phys. B388, 570 (1992). 
[28] R. Akhoury, R. Saotome, and G. Sterman, arXiv:1308.5204.

[29] K. Westpfahl, Fortschr. Phys. 33, 417 (1985).

[30] N. E. J. Bjerrum-Bohr, J. F. Donoghue, B. R. Holstein, L. Planté, and P. Vanhove, Phys. Rev. Lett. 114, 061301 (2015); J. High Energy Phys. 11 (2016) 117.

[31] L. D. Landau and E. M. Lifschits, in The Classical Theory of Fields: Course of Theoretical Physics, 4th ed. (ButterworthHeinemann, London, 1975), Vol. 2.

[32] B. M. Barker, M. S. Bhatia, and S. N. Gupta, Phys. Rev. 158, 1498 (1967); 162, 1750(E) (1967).
[33] I. J. Muzinich and S. Vokos, Phys. Rev. D 52, 3472 (1995).

[34] W. D. Goldberger and I. Z. Rothstein, Phys. Rev. D 73, 104029 (2006).

[35] J. B. Gilmore and A. Ross, Phys. Rev. D 78, 124021 (2008).

[36] S. Foffa and R. Sturani, Phys. Rev. D 84, 044031 (2011).

[37] L. Blanchet, Living Rev. Relativity 17, 2 (2014).

[38] S. Foffa, P. Mastrolia, R. Sturani, and C. Sturm, Phys. Rev. D 95, 104009 (2017).

[39] R. A. Porto, Phys. Rep. 633, 1 (2016). 